\title{
Evaluating the potential of post-processing kinematic (PPK) georeferencing for UAV-based structure- from-motion (SfM) photogrammetry and surface change detection
}

\author{
He Zhang ${ }^{1}$, Emilien Aldana-Jague ${ }^{1}$, François Clapuyt ${ }^{1}$, Florian Wilken ${ }^{2,3}$, Veerle Vanacker ${ }^{1}$, and \\ Kristof Van Oost ${ }^{1,4}$ \\ ${ }^{1}$ Earth and Life Institute, Georges Lemaître Centre for Earth and Climate Research, \\ Université Catholique de Louvain, Louvain-la-Neuve, 1348, Belgium \\ ${ }^{2}$ Institute for Geography, Universität Augsburg, Augsburg, 86159, Germany \\ ${ }^{3}$ Department of Environmental Systems Science, ETH Zurich, 8092 Zurich, Switzerland \\ ${ }^{4}$ Fonds de la Recherche Scientifique (FNRS), Brussels, 1000, Belgium
}

Correspondence: He Zhang (he.zhang@uclouvain.be)

Received: 9 January 2019 - Discussion started: 28 January 2019

Revised: 12 June 2019 - Accepted: 1 August 2019 - Published: 2 September 2019

\begin{abstract}
Images captured by unmanned aerial vehicles (UAVs) and processed by structure-from-motion (SfM) photogrammetry are increasingly used in geomorphology to obtain high-resolution topography data. Conventional georeferencing using ground control points (GCPs) provides reliable positioning, but the geometrical accuracy critically depends on the number and spatial layout of the GCPs. This limits the time and cost effectiveness. Direct georeferencing of the UAV images with differential GNSS, such as PPK (post-processing kinematic), may overcome these limitations by providing accurate and directly georeferenced surveys. To investigate the positional accuracy, repeatability and reproducibility of digital surface models (DSMs) generated by a UAV-PPK-SfM workflow, we carried out multiple flight missions with two different camera-UAV systems: a small-form low-cost micro-UAV equipped with a high field of view (FOV) action camera and a professional UAV equipped with a digital single lens reflex (DSLR) camera. Our analysis showed that the PPK solution provides the same accuracy (MAE: ca. $0.02 \mathrm{~m}$, RMSE: ca. $0.03 \mathrm{~m}$ ) as the GCP method for both UAV systems. Our study demonstrated that a UAV-PPK-SfM workflow can provide consistent, repeatable 4-D data with an accuracy of a few centimeters. However, a few flights showed vertical bias and this could be corrected using one single GCP. We further evaluated different methods to estimate DSM uncertainty and show that this has a large impact on centimeter-level topographical change detection. The DSM reconstruction and surface change detection based on a DSLR and action camera were reproducible: the main difference lies in the level of detail of the surface representations. The PPK-SfM workflow in the context of 4-D Earth surface monitoring should be considered an efficient tool to monitor geomorphic processes accurately and quickly at a very high spatial and temporal resolution.
\end{abstract}




\section{Introduction}

During the past decade, unmanned aerial vehicles (UAVs) or unmanned aerial systems (UASs) have emerged as a very valuable tool for aerial surveying (Passalacqua et al., 2015; Tarolli, 2014). An important application in geoscience is the generation of high-resolution topography (HRT) data (i.e., point clouds, digital surface models - DSMs - or digital elevation models - DEMs) from 2-D imagery using structure-from-motion (SfM) and multi-view stereo (MVS) photogrammetry (Eltner et al., 2016; James and Robson, 2012). Compared to satellite- or airborne-based sensing approaches, UAVs provide important advantages; more specifically, they provide a considerably higher spatial resolution at a relatively low cost in combination with high versatility in terms of sensors and data collection. With the capability of detecting topographical change at a very high resolution and accuracy, the UAV-SfM framework has become an increasingly used tool for the monitoring of landslides (e.g., Clapuyt et al., 2017; Turner et al., 2015), overland flow erosion (e.g., Eltner et al., 2017; Pineux et al., 2017), river dynamics (e.g., Hemmelder et al., 2018) and vegetation dynamics (e.g., Candiago et al., 2015).

However, the intercomparison of UAV-SfM photogrammetric products requires very accurate georeferencing. So far, the use of ground control points (GCPs) surveyed with precise GPS systems or total stations is generally employed for accurate positioning. The GCP-based georeferencing method has been widely proven to be a solid solution for accurate georeferencing (Hawkins, 2016; James et al., 2017; Turner et al., 2016). However, GCPs need to be placed as a network, and this comes at a cost as it is time-consuming. Furthermore, the accuracy depends on the quantity and distribution of GCPs (Sanz-Ablanedo et al., 2018). When used in a monitoring study, additional issues arise from the fact that GCPs can move (weather impact or surface deformations). Finally, a major limitation arises from the fact that GCPs cannot be placed in poorly accessible terrain due to practical or safety reasons (e.g., swamps, landslides or glaciated areas).

Direct georeferencing based on high-precision GNSS is key to overcoming this issue, but it requires the accurate geotagging of aerial images at the exposure time. During the last several years, the development of high-quality inertial measurement unit (IMU) and global navigation satellite system (GNSS) technology as well as dedicated RTK (real-time kinematic) and PPK (post-processing kinematic) solutions for UAVs has enabled the accurate measurement of UAVcamera position and orientation. By double differencing the phase ambiguities between two GNSS-GPS receivers, atmosphere propagation delay and receiver clock errors can be eliminated. RTK positioning requires a stable radio (or internet) link between a base and the UAV, and this can sometimes be challenging due to radio link outages and/or GNSS signal blocks. PPK, in contrast, processes the information after the flight and there is thus no risk of data loss due to link outages. In addition, precise ephemeris data of GNSS satellites are available during post-processing, which can often provide a more accurate solution. The utilization of such an approach has the potential to avoid or mitigate the need for GCPs. Several studies already investigated the application of RTK-PPK direct georeferencing by the integration of sensor orientation with onboard RTK GPS (Fazeli et al., 2016; Forlani et al., 2018; Stöcker et al., 2017). In a study performed by Gerke and Przybilla (2016), block orientation accuracy was significantly enhanced by using an onboard RTK GNSS solution. With an enabled RTK GNSS and cross-flight pattern, the best scenario reached a final horizontal geometric accuracy of $4 \mathrm{~cm}$. Recently, both georeferencing methods have gradually matured and can deliver centimeter-level accuracy in geomorphological applications (Table 1). However, to our knowledge the accuracy and repeatability of HRT products derived from RTK-PPK in the context of longerterm 4-D Earth surface monitoring with time-lapse structurefrom-motion photogrammetry has not been quantified.

The accuracy and precision of photogrammetry depends on many other factors, including image quality, camera calibration, flight plan characteristics, SfM algorithms, and surface texture and albedo. The bundle block adjustment (BBA) process determines the 3-D positions of key features and points presented in the overlapping part of multiple images by recognizing and matching key points (hereafter referred to as tie points, i.e., key points that can be identified on two or more images). In a next step the relative locations and orientations of the camera are estimated by performing a fit and minimizing the error through the tie points (Triggs et al., 2000). The abovementioned factors affect the identification of the tie points, which are infrequently reported but important nevertheless. Therefore, the accuracy of traditional photogrammetric data depends heavily on control quality, whereas SfM accuracy is also strongly affected by image characteristics (Mosbrucker et al., 2017).

The selection and configuration of cameras are of special interest in UAV photogrammetry. Digital cameras equipped with high-quality sensors (e.g., a DSLR camera) provide better image quality due to higher resolution and reduced image noise relative to more portable and smaller sensors (e.g., a compact or action camera), and this results in high-quality DSMs (Eltner and Schneider, 2015; Micheletti et al., 2015; Mosbrucker et al., 2017). The focal length relates to radial distortion and associated calibration of the camera lens (Rosnell and Honkavaara, 2012; Sanz-Ablanedo et al., 2012). While small focal length (or wide angle) leads to a large field of view (FOV), which therefore requires a less dense flight plan for a given lateral overlap, these images are subject to increased radial distortion, which can degrade accuracy (James and Robson, 2014; Mosbrucker et al., 2017). Some studies have investigated the impact of focal length on DEM accuracy (Clapuyt et al., 2016) but mainly on DEM reproducibility. Furthermore, the distance between the sensor and the surface also determines ground sample distance 


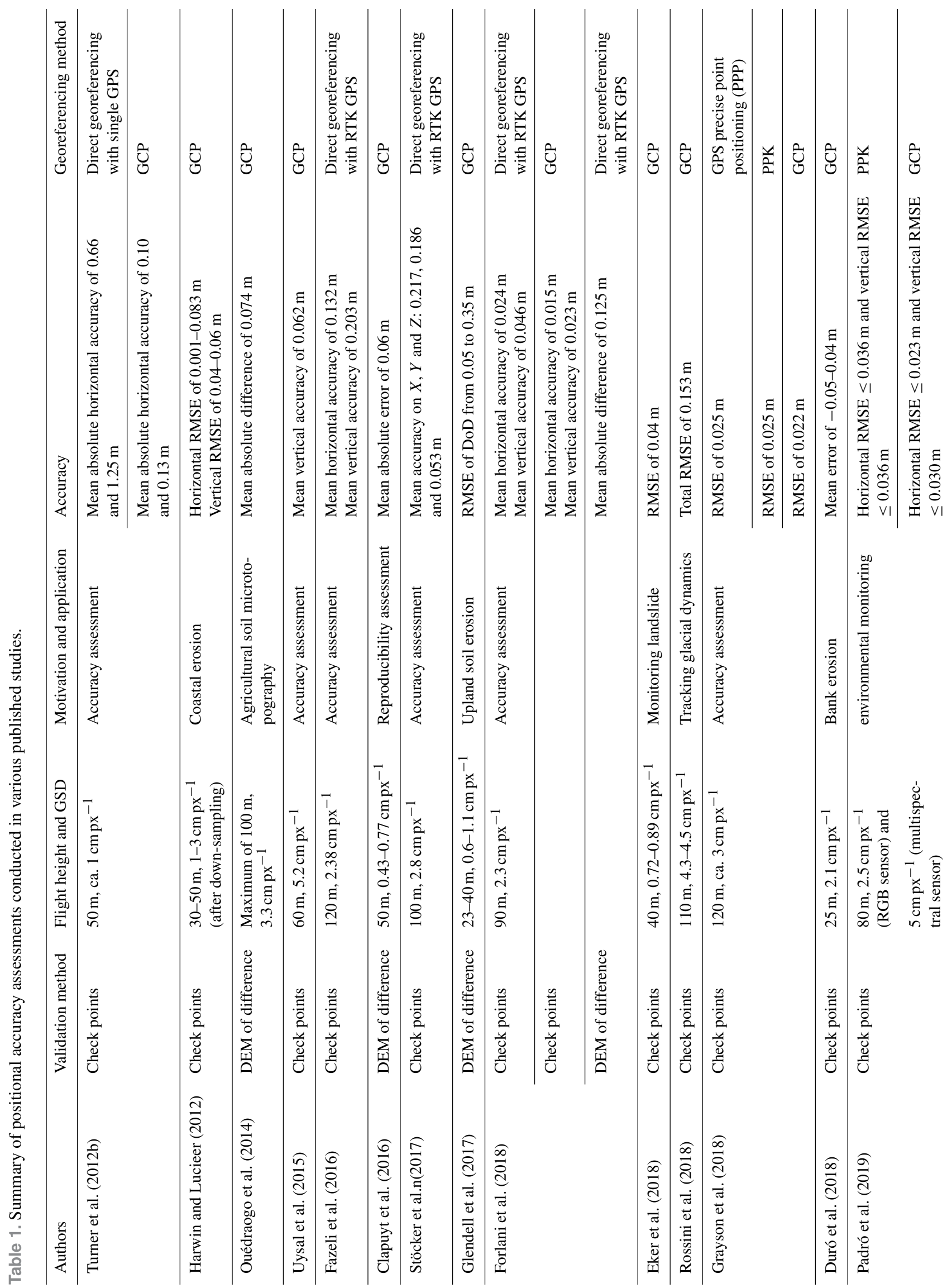


(GSD), which impacts accuracy. Eltner et al. (2016) showed in a review of 54 studies that the error of SfM-derived DSMs increased nonlinearly with an increasing surface to camera distance (Eltner et al., 2016). From an operational point of view, camera weight is a critical variable as it determines the size and weight of the UAV system. There is a large difference in weight between DSLR $(0.5-1.5 \mathrm{~kg})$ and action cameras $(0.05-0.15 \mathrm{~kg})$, and this has large implications, not only for flight autonomy (and hence spatial coverage), but also the choice of the UAV platform. Small action cameras can be mounted on small "micro-drones", which are subjected to less stringent UAV flight regulation (e.g., in Belgium, a UAV operation certificate allows for a maximum flight height of $45 \mathrm{~m}$ and a weight limit of $5 \mathrm{~kg}$, UAV + payload).

The quality of UAV survey output is typically analyzed using the spatial patterns of errors in DSMs, and this includes both the accuracy and the reproducibility of DSM generation. Errors propagate when differences of DSMs (DEM of differences, DoDs) are computed to quantify topographic change. Given the uncertainty inherent in individual DSMs, how to distinguish real geomorphic changes from noise and how well these uncertainties are considered control the reliability of interpretation. In order to isolate and quantify the uncertainty that is associated with the topographic reconstructions, reproducibility assessments are critical aspects of monitoring landform changes over time (Brasington et al., 2000; Wheaton et al., 2010). However, until now the repeatability of direct PPK-based georeferencing for SfM-derived point clouds and/or DSMs has not been thoroughly evaluated. Past research has shown that a RTK-SfM workflow is repeatable (Forlani et al., 2018), but the analysis was based on repeated flights conducted over a very short time frame: i.e., with very similar satellite constellation, base station setup and light conditions. It remains uncertain to what extent a PPKSfM workflow may provide consistent 4-D data when survey conditions are variable, e.g., when monitoring over longer periods of time (e.g., weeks or even months). This is particularly relevant for geomorphological applications that require centimetric precision such as rill erosion or soil roughness monitoring (d'Oleire-Oltmanns et al., 2012; Eltner et al., 2015). A second issue is the platform: low-cost, easily deployable, RTK-enabled micro-UAVs (small form ca. $25 \times 25 \mathrm{~cm}$, weight $1.4 \mathrm{~kg}$ ) equipped with small cameras have recently become available, but their accuracy and repeatability, relative to professional UAV systems (large form ca. $80 \times 80 \mathrm{~cm}$, weight $4.5 \mathrm{~kg}$ ) equipped with high-end cameras, remain poorly quantified. In particular, the influence of the UAV-camera setup on the minimum level of topographical change detection should be quantified in order to guide geomorphological applications.

The main objective of this study is thereby to quantify the (i) repeatability, (ii) reproducibility and (iii) efficiency of the PPK-SfM framework in the context of 4-D Earth surface monitoring with time-lapse structure-from-motion photogrammetry, for which centimetric precision is required.

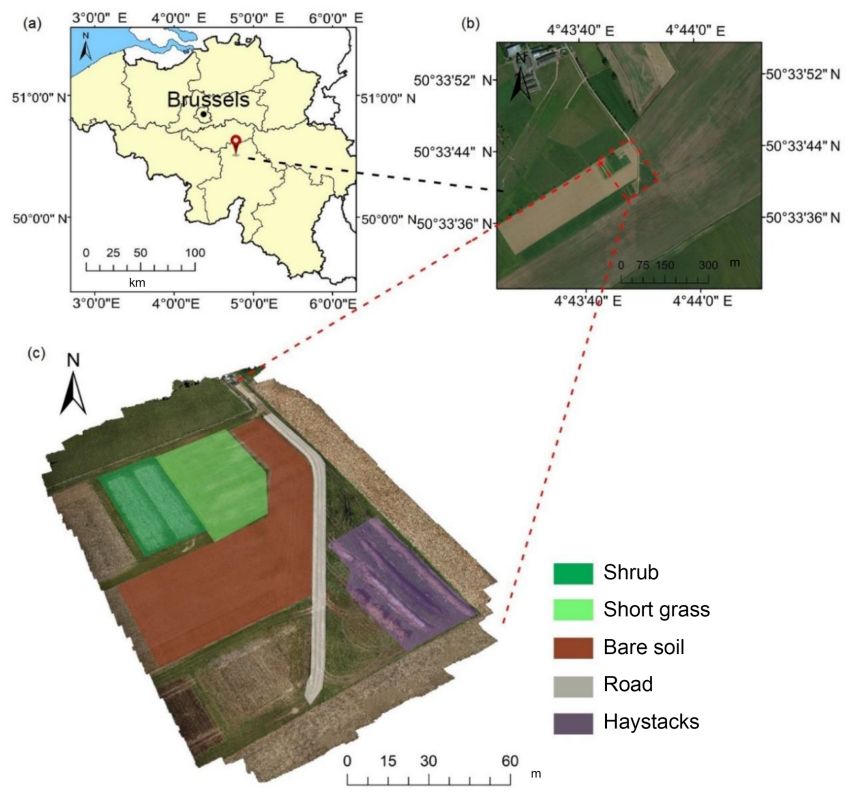

Figure 1. Description of the study sites. (a) Location of the study site, (b) satellite image of the study site and (c) classification of the surface used in the analysis. Note: (a) and (b) are taken from (C) Google Maps, and (c) is produced by UAV imagery.

More specifically, we aim to (i) assess the accuracy and repeatability of PPK and non-PPK solutions in georeferencing to examine the capability of using PPK without the need for GCPs, (ii) assess the reproducibility of surface topography change detection using PPK solutions for two different UAV-camera setups (i.e., a DSLR camera versus a high-FOV action camera), and (iii) evaluate different approaches to estimate uncertainties using PPK solutions and their implications for surface change detection.

\section{Material and methods}

\subsection{Study site}

The study site is located in an agricultural area (1.7 ha) in the Belgium loess belt ca. $40 \mathrm{~km}$ southeast of Brussels, Belgium (Fig. 1). It is characterized by a slightly undulated terrain with an altitude range between 207 and $210 \mathrm{~m}$ a.s.l. and by very gentle slopes (mean slope: $1^{\circ}$ ). The site is partially cultivated, while other parts are covered by grass. The surface was classified into five classes: i.e., bare soil, short grass, shrub, road and haystacks.

\subsection{Hardware setup}

\subsubsection{Platforms and payloads}

We evaluated (i) a high-payload UAV system equipped with a DSLR camera and (ii) a consumer-grade UAV equipped with a fish-eye action camera. The high-payload aerial sys- 
tem is a custom-built Hexacopter and is equipped with a DJI A2 flight controller. The platform has an effective payload of $4 \mathrm{~kg}$ and an autonomy of ca. $15 \mathrm{~min}$. This UAV was equipped with a Canon EOS 550D camera (18 megapixels, $5184 \times 3456$ pixels, with Canon EF $28 \mathrm{~mm}$ F/2.8 lens). The consumer-grade UAV was a DJI Phantom 3 Advanced Drone. We removed the DJI camera-gimbal system and mounted a GoPro Hero 3 camera (12 megapixels, $4000 \times$ 3000 pixels, with $2.92 \mathrm{~mm} \mathrm{~F} / 2.8123^{\circ} \mathrm{HFOV}$ lens) (Fig. 2). Both platforms are equipped with a compact multi-GNSS RTK receiver (Reach RTK kit, Emlid Ltd) with RTK-PPK capability as described below.

\subsubsection{PPK GPS module}

During the UAV flights, a Reach RS (Emlid Ltd) base station was mounted on a tripod located in the north of the test area to provide positioning correction input. The maximal distance between the UAV and the base station was $220 \mathrm{~m}$. The receiver of the base is configured to log the raw data in a RINEX file at $5 \mathrm{~Hz}$ using the satellite GPS, GLONASS and GALILEO. We did not use a fixed position for the base station but randomly positioned it in an area of ca. $10 \times 10 \mathrm{~m}$ for each flight. Both UAVs were equipped with a Reach GNSS receiver to log the raw data as UBX format using GPS and GLONASS satellites. The antenna model was a Tallysman TW2710, which covers the GPS L1, GLONASS G1, BeiDou B1, Galileo E1 and SBAS (WAAS, EGNOS and MSAS) frequency bands. The antenna was mounted on an aluminum plate, with the center right above the camera lens center to minimize the offset shift between the antenna phase center and camera projection center. The antenna height was $22.5 \mathrm{~cm}$, and this difference between the antenna and camera projection center was considered during the post-processing. No lever-arm corrections were considered, but the offset between the camera and the GPS receiver was considered in the camera position assuming a constant vertical offset (see below). Because of the small magnitude of the physical offset vector $(0,0$ and $22.5 \mathrm{~cm}$ in $X, Y$ and $Z$ in the body frame, respectively), typical tilting during flights would only propagate to a camera position error of about $1 \mathrm{~cm}$, which is close to the expected GPS positioning error of about $2-3 \mathrm{~cm}$.

For the high-payload UAV, we used the hot shoe of the camera to time-mark the pictures with a GPS event logged on a Reach GNSS device mounted on the UAV. As the action camera has no hot shoe, we built an electronic system to integrate and synchronize the GPS with the action camera. To this end, a single-board computer (SBC) is used as a trigger by transmitting an electrical signal to both the camera and GPS unit. To eliminate the lag between the shutter opening time of the camera and the GPS recording time, we quantified the delay between the electrical signal and the shutter opening by integrating an LED light in the circuit. Several delay times were tested until the LED light was visible on the images taken by the action camera. This procedure resulted in a system in which the geotagging was accurately synchronized with the GPS time. For both UAV-camera systems, we did not build a link between the UAV-IMU and camera. As a result, the images only contained positioning information without attitude parameters.

\subsection{Data collection \\ 2.3.1 Flight planning}

Flight missions were planned using the Autopilot app (Hangar Technology, 2018). The side overlap was set to $80 \%$. The frontal overlap was defined by the speed of the $\mathrm{UAV}$ and the camera trigger interval, which was set at $2 \mathrm{~s}$ for the DSLR camera and $4 \mathrm{~s}$ for the action camera; this resulted in a frontal overlap of ca. $90 \%$ for both systems.

Flight mission arrangements are summarized in Table 2. Three flights (including repeated flights) were conducted before a part of the study area was plowed. These flights were conducted at a constant height above the take-off point, leading to a ground sample distance (GSD) of less than 0.63 and $3.11 \mathrm{~cm}$ for the DSLR and the action camera, respectively. It should be noted that the missions were performed using a simple parallel rather than cross-hatch flight pattern, as the latter mission setup can mask systematic bias.

\subsubsection{Ground control points}

A total of 16 fixed targets were distributed evenly across the study area before the survey as control points (Fig. 2). Depending on the georeferencing methods used (see below), the control points were applied as ground control points (GCPs) or check points (CPs). The targets consisted of a laminated square board $(0.3 \mathrm{~m} \times 0.3 \mathrm{~m})$ painted in yellow and a black cross marker in center. They were fixed with nails into the ground and remained at the site for the study period before plowing. For the last flight mission after plowing, new GCPs were deployed and surveyed. The targets were surveyed after each flight mission using a Reach RS (RTK solution) with the EUREF-IP network. The correction stream was provided by BRUS station (Brussels, Belgium; antenna: ASH701945B_M) via NTRIP (Networked Transport of RTCM via Internet Protocol), which had a mean planimetric error of $0.007 \mathrm{~m}$ and altimetric error of $0.013 \mathrm{~m}$ (https://emlid.com/, last access: 1 January 2019). Based on repeated measurements of field GCP coordinates, the planimetric precision was estimated at $0.015 \mathrm{~m}$, while the altimetric precision was $0.023 \mathrm{~m}$. It should be noted that this assessment includes minor $(\mathrm{G}) \mathrm{CP}$ movement induced by rainfall kinetic energy and soil swelling and shrinking. The coordinate system was referenced to the World Geodetic Datum of 1984 (WGS84). 

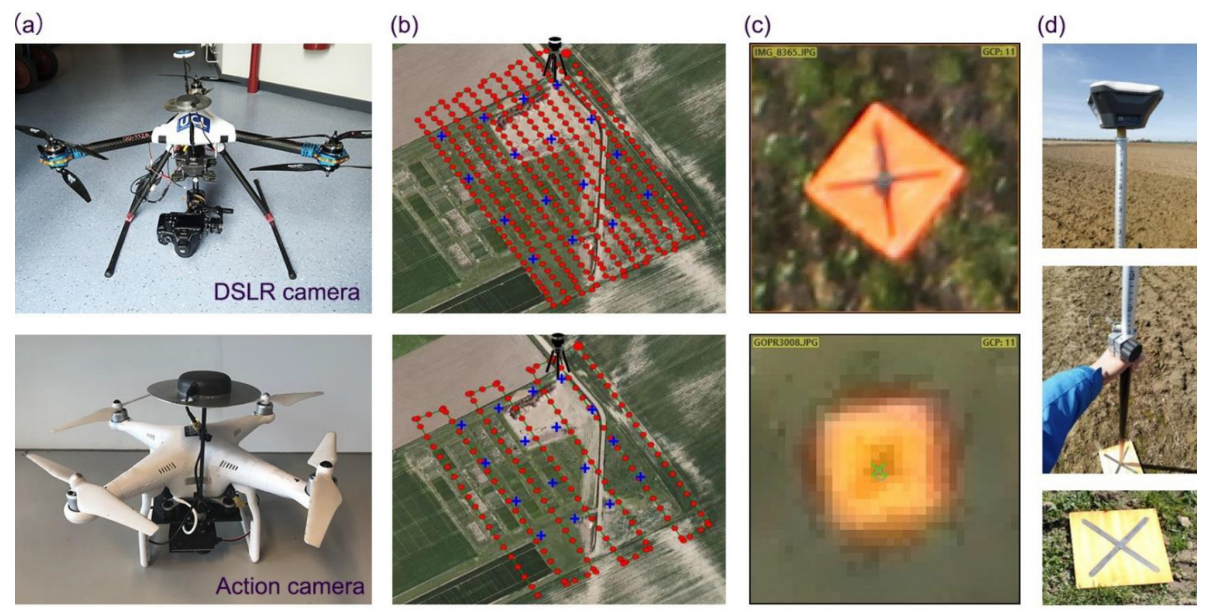

Camera locations

t GCP/CP locations

기

Base station

Figure 2. Experimental setup. (a) UAV-camera setup: DSLR camera (EOS 550D) mounted on RPAS type Y6, action camera (GoPro Hero 3) mounted on a Phantom 3 Advanced. (b) Parallel flight lines (top: RPAS type Y6 with DSLR camera; bottom: Phantom 3 with action camera) and GCP-CP distribution (c). How GCPs-CPs are displayed in the images and (d) measurement of GCPs-CPs.

Table 2. Overview and key parameters of flight missions.

\begin{tabular}{|c|c|c|c|c|c|c|c|c|c|}
\hline & Camera & Date & $\begin{array}{l}\text { Mission } \\
\text { number }\end{array}$ & $\begin{array}{r}\text { Flight } \\
\text { height } \\
\text { (m) }\end{array}$ & $\begin{array}{r}\text { Speed } \\
\left(\mathrm{m} \mathrm{s}^{-1}\right)\end{array}$ & $\begin{array}{r}\text { Area } \\
\text { covered } \\
\text { (ha) }\end{array}$ & $\begin{array}{r}\text { Satellite } \\
\text { PDOP } \\
\text { value }\end{array}$ & $\begin{array}{r}\text { Ground } \\
\text { sampling } \\
\text { distance } \\
\left(\mathrm{cm} \mathrm{px}^{-1}\right)\end{array}$ & $\begin{array}{r}\text { Number } \\
\text { of } \\
\text { images }\end{array}$ \\
\hline \multirow[t]{6}{*}{ Before plowing } & DSLR & 29 March 2018 & $\mathrm{~F} 1$ & 45 & 3.4 & 3.75 & 1.3 & 0.6 & 323 \\
\hline & camera & 5 April 2018 & F2_a & 45 & 3.4 & 3.26 & 1.2 & 0.6 & 360 \\
\hline & $(\mathrm{EOS})$ & & F2_b & 45 & 3.4 & 3.26 & 1.2 & 0.6 & 362 \\
\hline & Action & 29 March 2018 & F1_a & 45 & 3.4 & 11.33 & 1.3 & 3.1 & 134 \\
\hline & camera & & F1_b & 45 & 3.4 & 13.27 & 1.2 & 3.1 & 155 \\
\hline & (GoPro) & 30 March 2018 & $\mathrm{~F} 2$ & 45 & 3.4 & 12.05 & 1.4 & 3.1 & 137 \\
\hline \multirow[t]{4}{*}{ After plowing } & DSLR camera & 6 April 2018 & F3_a & 35 & 3.0 & 0.85 & 1.3 & 0.5 & 129 \\
\hline & $(\mathrm{EOS})$ & & F3_b & 35 & 3.0 & 0.8 & 1.2 & 0.5 & 107 \\
\hline & Action camera & 6 April 2018 & F3_a & 20 & 2.6 & 3.23 & 1.2 & 1.3 & 182 \\
\hline & (GoPro) & & F3_b & 20 & 2.6 & 3.01 & 1.2 & 1.3 & 162 \\
\hline
\end{tabular}

Note: repeated flight missions were marked as F_a and F_b. The missions shown in the list used a parallel flight plan.

\subsection{Data processing}

\subsubsection{Georeferencing configuration}

The open-source software package RTKLib was used for computing differential positioning (Takasu and Yasuda, 2009). Raw GPS data from the UAV-mounted cameras and the base station were then extracted and corrected by postprocessing using RTKLib. We verified the consistency of the estimated camera positions using PPK by evaluating different satellite elevation masks $\left(15\right.$ and $\left.20^{\circ}\right)$ and methods (i.e., "fix-and-hold" versus continuous mode).
We extracted PPK GPS and single GPS solutions for the camera position estimates. To assess the accuracy of different georeferencing options, datasets were processed with four configurations, i.e., single GPS, single GPS +GCPs, PPK only and $P P K+1 G C P$. For the conventional methods using GCPs and a single GPS, we used the RTKLib single GPS solution to acquire the image coordinates and selected half of the targets as 3-D GCPs during block control processing. The remaining control points were then used as check points. The setup of GCP-CP is shown in Fig. 3. In the single $G P S+G C P s$ scenario, the eight selected GCPs were evenly distributed in the survey area. In the $P P K+1 G C P$ scenario, 
cross-validation was used. We selected one point as a GCP, while the remaining targets were then used as CPs, and this bundle adjustment processing was repeated 16 times. The accuracy assessment was based on the average error of the cross-validation.

\subsubsection{Point cloud and DSM generation}

The geotagged images were processed with the Pix4D Mapper software (https://www.pix4d.com/, last access: 8 August 2019). The software uses the SfM algorithm to generate 3-D point clouds, DSMs and orthophoto mosaics of the surveyed area. The procedure consists of three main steps: (i) initial processing, (ii) point cloud generation, and (iii) DSM and orthomosaic generation. First, the photographs are aligned using a point matching algorithm that automatically detects matching points on overlapping photographs and uses these points to simultaneously solve for exterior orientation (EO) parameters. With additional position information that is available for the images or GCPs, the software then georeferences the model and refines the camera calibration by minimizing the error between the modeled locations of the points and the measured locations; meanwhile, nonlinear deformations within the model are corrected.

Camera accuracy is a key parameter allowing users to set how accurate the coordinates of images can be, which would affect the determination of estimated camera positions in the BBA process. Considering the precision of PPK GPS (ca. $0.02 \mathrm{~m}$ ) and the antenna angle movement caused by UAV attitude during flying, we set both the horizontal and vertical accuracy as $0.05 \mathrm{~m}$. We used the Pix 4D 3-D map template for the remaining settings, i.e., a full key point image scale, an automatic targeted number of key points and a standard calibration method. In order to maintain the characteristics of the original data, the clouds were not filtered or smoothed. Gridded DSMs were then generated based on the mean altitude of these point clouds. The 3-D outputs (i.e., point clouds and DSMs) used for reproducibility assessment were georeferenced using the PPK method (and no GCPs were considered). The corresponding grid resolutions of the DSMs were less than $0.031 \mathrm{~m}$ for the action camera and $0.006 \mathrm{~m}$ for the DSLR camera.

\subsection{Data analysis}

\subsubsection{Accuracy assessment}

Absolute accuracy validation was performed using the CPs (which were not used in the BBA process) by comparing the coordinates of the 16 CPs in the 3-D cloud with the reference values measured in the field by RTK GNSS. The mean absolute error (MAE), the root mean square error (RMSE) and standard deviation of the differences were computed for each flight to (i) assess the accuracy of SfM outputs with different georeferencing configurations, (ii) assess the precision of PPK-SfM reconstruction considering CPs as static references during the observation period (i.e., with variable satellite constellation, light conditions and base station setup), and (iii) detect whether there are internal systematic shifts and block deformations in the SfM output.

\subsubsection{Precision maps based on Monte Carlo simulation}

To demonstrate how tie point uncertainty can vary spatially, we implemented a Monte Carlo approach that enabled precision maps to be produced when using SfM-based software. Following the workflow by James et al. (2017), the processing was implemented using a combination of PhotoScan Professional (v1.2.4; for image processing and bundle adjustment), Python (integrated into PhotoScan for Monte Carlo execution) and sfm_georef (v3.1; James and Robson, 2012, for visualization of results). To construct the image network, images were automatically matched and oriented in PhotoScan using the "align images" function. During the alignment process, the georeferencing was achieved by PPK positioning camera coordinates without GCP reference. The subsequent Monte Carlo analyses were carried out in PhotoScan using a Python script to automate repeated bundle adjustments. The simulated pseudo-random error (camera accuracy) was set as $0.05 \mathrm{~m}$ considering the precision of PPK GPS and the antenna movement caused by drone attitude. The Monte Carlo processing comprised 1000 iterations for each survey. Afterwards, the results from all iterations are compiled to give distributions of determined values for all estimated parameters (e.g., coordinate values for each sparse point). To construct 3-D precision maps, point coordinate standard deviations in $X, Y$, and $Z$ directions are calculated for each point and interpolated onto a grid, generating a raster map representing the spatially variable precision of tie points. For both camera datasets, we obtained precision maps for each survey and compared their range with $\mathrm{CP}$ observation precisions (i.e., precision of $\mathrm{CP}$ residuals) from the repeated surveys by extracting values from corresponding $\mathrm{CP}$ positions.

\subsubsection{Repeatability and reproducibility assessment}

To robustly distinguish real changes in DSM-DEM differencing from the inherent noise (Fuller et al., 2003), DoD uncertainty must be considered. Regardless of the approach used to generate DSM-DEMs, the process of accounting for DoD uncertainty follows a consistent progression via three steps: (i) quantifying the error surface $(\delta z)$ of each individual DSM surface, (ii) propagating the identified uncertainties into the $\operatorname{DoD}\left(\delta u_{\mathrm{DoD}}\right)$ and (iii) assessing the significance of the propagated uncertainty (Wheaton et al., 2010). The tie points differ between each repetition of the survey, and therefore we analyze the error propagation at the DSM level. There are two primary ways to build an error surface. The combined error can be calculated as a single value for the 

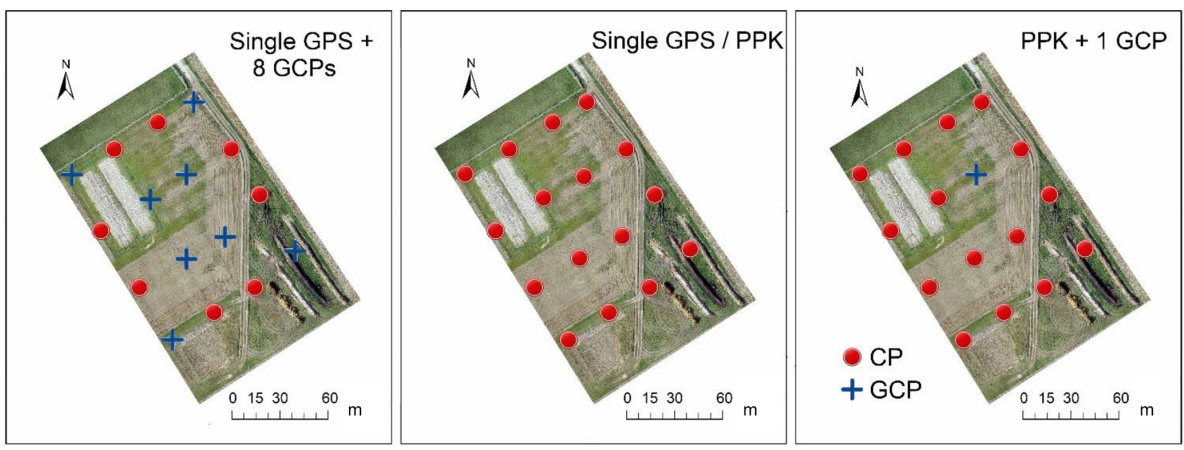

Figure 3. Distribution of GCPs and CPs and illustration of the different georeferencing configurations: single GPS, single GPS +8 GCPs, $P P K, P P K+1 G C P$. Note: cross-validation was implemented in the $P P K+G C P$ configuration; i.e., one single control point was used as a GCP in each processing.

entire DoD based on the average RMSE of each DEM if spatially explicit estimates of the error do not exist. This method assumes that the errors in each cell are random and independent. Alternatively, a spatially variable error can be considered for both DEMs independently (e.g., Wheaton et al., 2013). The individual error in the DSMs can be propagated into the $\mathrm{DoD}$ as

$\delta u_{\mathrm{DoD}}=\sqrt{\left(\delta z_{\text {ref }}\right)^{2}+\left(\delta z_{\mathrm{comp}}\right)^{2}}$,

where $\delta u_{\mathrm{DoD}}$ is the propagated error in the DoD as a minimum level of detection threshold $\left(\mathrm{LoD}_{\min }\right)$, and $\delta z_{\text {ref }}$ and $\delta z_{\text {comp }}$ are the individual error in the referenced DSM and compared DSM, respectively.

To define a spatially variable confidence interval associated with each measurement and combining the uncertainties, a prescribed confidence level ( $95 \%$ in the following) is used to locally estimate the measurement accuracy and precision. The registration error $(r e g)$ is considered and assumed isotropic and spatially uniform, as there may be systematic bias in georeferencing (e.g., Brasington et al., 2003; Wheaton et al., 2013). Equation (1) can be hereby modified to

$\operatorname{LOD}_{95 \%}= \pm 1.96\left(\sqrt{\left(\delta z_{\mathrm{ref}}\right)^{2}+\left(\delta z_{\mathrm{comp}}\right)^{2}}+r e g\right)$,

where reg is the relative overall registration error between the surveys. $\mathrm{LoD}_{95} \%$ is the level of detection at the $95 \%$ confidence interval.

\subsubsection{UAV-based monitoring of surface change}

As mentioned above, the farmland was plowed on 6 April, leading to surface roughness and volume change. Surveys implemented before and after the plowing were compared to detect the change. In this case study, the $P P K$ dataset was potentially subjected to higher reg error, while the $P P K+1 G C P$ scenario might be able to substantially mitigate the reg error. Therefore, a reg value was used based on the CP RMSEs for the $P P K$ dataset, whereas the
$P P K+1 G C P$ dataset was regarded with negligible reg error. In that case, an assessment of the constant and spatialized LoD was carried out: a DoD using a survey-wide LoD based on the $Z$ RMSE on CPs and a spatialized LoD based on the Monte Carlo altimetric precision. A zoomed-in area as well as a transect was sampled to illustrate the surface change using the $\mathrm{LoD}_{\min }$ thresholds (Fig. 6). The sediment budget was subsequently assessed using the Geomorphic Change Detection (GCD) software (Wheaton et al., 2010). The GCD software provides the capability of segregating and quantifying uncertainties independently in each DEM and propagating them through to the DEM of difference. For each DEM, we set the two previously mentioned uncertain surfaces in the change detection between surveys.

\section{Results}

\subsection{Accuracy and precision of the georeferencing methods}

Table 3 summarizes the average (i.e., considering all the flights) check point accuracy and precision ranges in the $X$, $Y$ and $Z$ directions for each block control configuration. For the DSLR surveys, the single GPS configuration provided an average planimetric and altimetric RMSE of 1.59 and $3.45 \mathrm{~m}$, respectively, while the RMSEs for the other three georeferencing configurations were all below $0.036 \mathrm{~m}$. For $P P K$, the altimetric CP RMSE was $0.036 \mathrm{~m}$, and the average was only slightly (ca. $20 \%$ ) improved when adding one GCP. For the action camera, the CP RMSEs for the GCP solution are better than 1 pixel for the $X, Y$ and $Z$ coordinates. The CP RMSEs for the $P P K$ solutions were slightly higher than for the $G C P$ solution but in the range of 0.5-1.4 pixels for the $X, Y$ and $Z$ coordinates. The values reported here are very close to the estimated error of the $P P K$ solution. When using single $G P S+G C P$, the accuracy was substantially enhanced to the centimeter level, and the absolute mean errors were less than $0.028 \mathrm{~m}$. For both cameras, $P P K+1 G C P$ showed similar planimetric accuracy and better altimetric accuracy. 
Adding one GCP improved the accuracy of the altimetric CP RMSE of the $P P K$ solution by $20 \%$ to $30 \%$. The standard deviation for the mapping errors (SD error) is very similar for both cameras and the PPK and GCP solutions (0.02-0.04 m), while as expected, the precision was not as good for the single solution $(0.24-0.35 \mathrm{~m})$.

\subsection{PPK accuracy and repeatability}

Figure 4 shows the $\mathrm{CP}$ residual distributions for each survey for the $P P K$ solution. As reported above, the overall accuracies and precisions among the surveys were robust within a range of $0.10 \mathrm{~m}$, regardless of dates and missions. The planimetric accuracies of the surveys were robust (with little bias) and the errors were close to zero. In contrast, the altimetric accuracy showed a much higher uncertainty among the surveys, with substantial bias for some flights. Similar results were obtained for both camera setups; i.e., altimetric errors showed larger variation than planimetric errors. We can also express the accuracies in pixels to standardize the RMSEs in terms of the expected error incurred from GSD. The CP $X Y Z$ RMSEs for the DSLR camera correspond to ca. 4-15 pixels. However, it should be noted that the GSD for the DSLR camera is extremely fine $(0.006 \mathrm{~m})$ due to the low flight height, and this is much finer than the width of the markers used on the CP $(0.02 \mathrm{~m})$ or the precision of the CPs. As a result, the $X Y Z$ RMSEs for the action camera were better and within a range of 1 to 5 pixels.

\subsection{PPK precision}

For soil surface change detection, it is important to quantify the precision of each surface. Here, we compare different methods to quantify precision. Figure 5 shows tie point precision maps derived from the Monte Carlo (MC) simulations. Spatial patterns can be observed from the DSLR precision map, where shrub areas have higher uncertainties, and non-vegetated areas were modeled more precisely. The DSLR dataset had a much better precision and smaller range ( 0 to $0.05 \mathrm{~m}$ ) when compared to the MC simulations for the action camera dataset. For the action camera, the precision ranged between 0 and $0.25 \mathrm{~m}$. In contrast to the results obtained for the DSLR camera, the precision map for the action camera did not show a clear structured spatial pattern. The box plots represent the CP-derived precision based on the five repeated surveys (16 CPs were used in each survey) (Fig. 5c and d). The DSLR precision maps derived from the MC simulations are in line with the empirical precision derived from the CPs (i.e., 0.01 to $0.03 \mathrm{~m}$ ). The slightly higher mean and range obtained for the empirical precision reflects the fact that for the MC analysis, only uncertainty in camera position was considered, while the empirical estimates reflect all sources of variability (i.e., positioning uncertainties, differences in image quality between surveys, etc.). In contrast, the action camera MC precision was substantially 


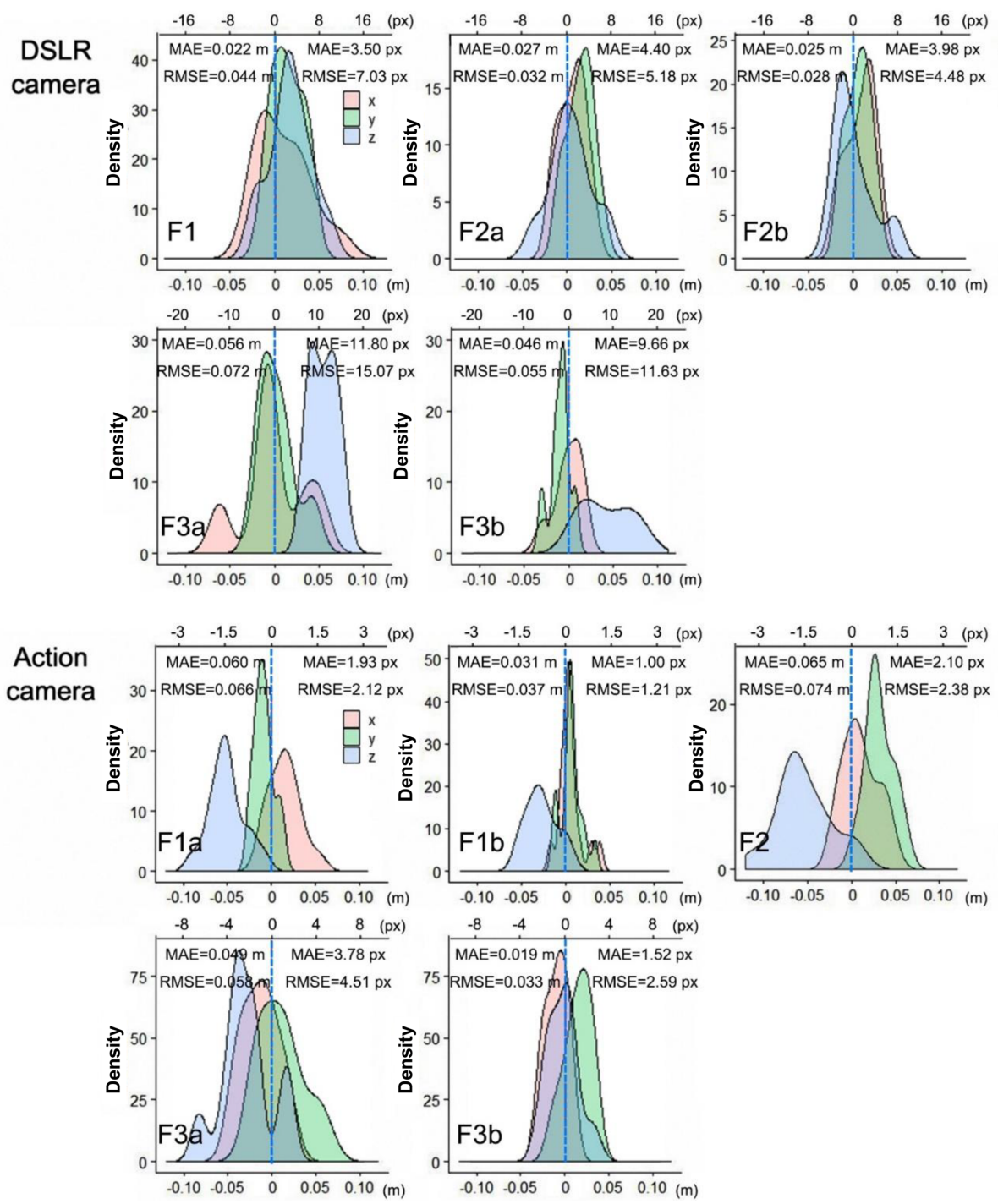

Figure 4. Distribution of CP residuals on the $X, Y$ and $Z$ directions of each survey. MAE and RMSE in the legend indicate mean absolute error and root mean square error of the $X Y Z$ direction. Units are given in meters as well as pixels to standardize results in terms of the expected error incurred from the GSD at the corresponding flight height.

higher than the precision derived from the repeated CP surveys. In other words, the observational precision estimates were smaller than those estimated from the MC analysis.

Based on the MC precision maps, spatially propagated error estimates can be generated for the repeated surveys (Fig. A1). The spatially distinct errors can be quantified: shrubs had a larger error of detection $(0.031 \mathrm{~m})$. The distribution of errors also showed lower precision for shrubs. For the rest of the surface types, the MC precision was around $0.02 \mathrm{~m}$. For the action camera dataset, no clear spatial pattern was found, and a spatially uniform precision is therefore a good approximation.

\subsection{Soil surface change detection}

In order to illustrate the potential of PPK in high-resolution surface change detection, we evaluate various approaches and camera setups. At the end of the monitoring period, the surface of the study area changed substantially as a result of plowing. The DSMs of the plowed area (before and after plowing) were analyzed (Fig. 6). For the $P P K$ datasets, when the mean $X Y Z$ RMSE was used to estimate the registration error, the threshold was high and substantially reduced the sensitivity in change detection. For the $P P K+1 G C P$ datasets, we observed that the bias (particularly in the $Z$ direction) was removed, and we therefore set the registration error to zero. We then applied both a spatially uniform DoD threshold (based on the CP RMSEs) and a spatially variable 

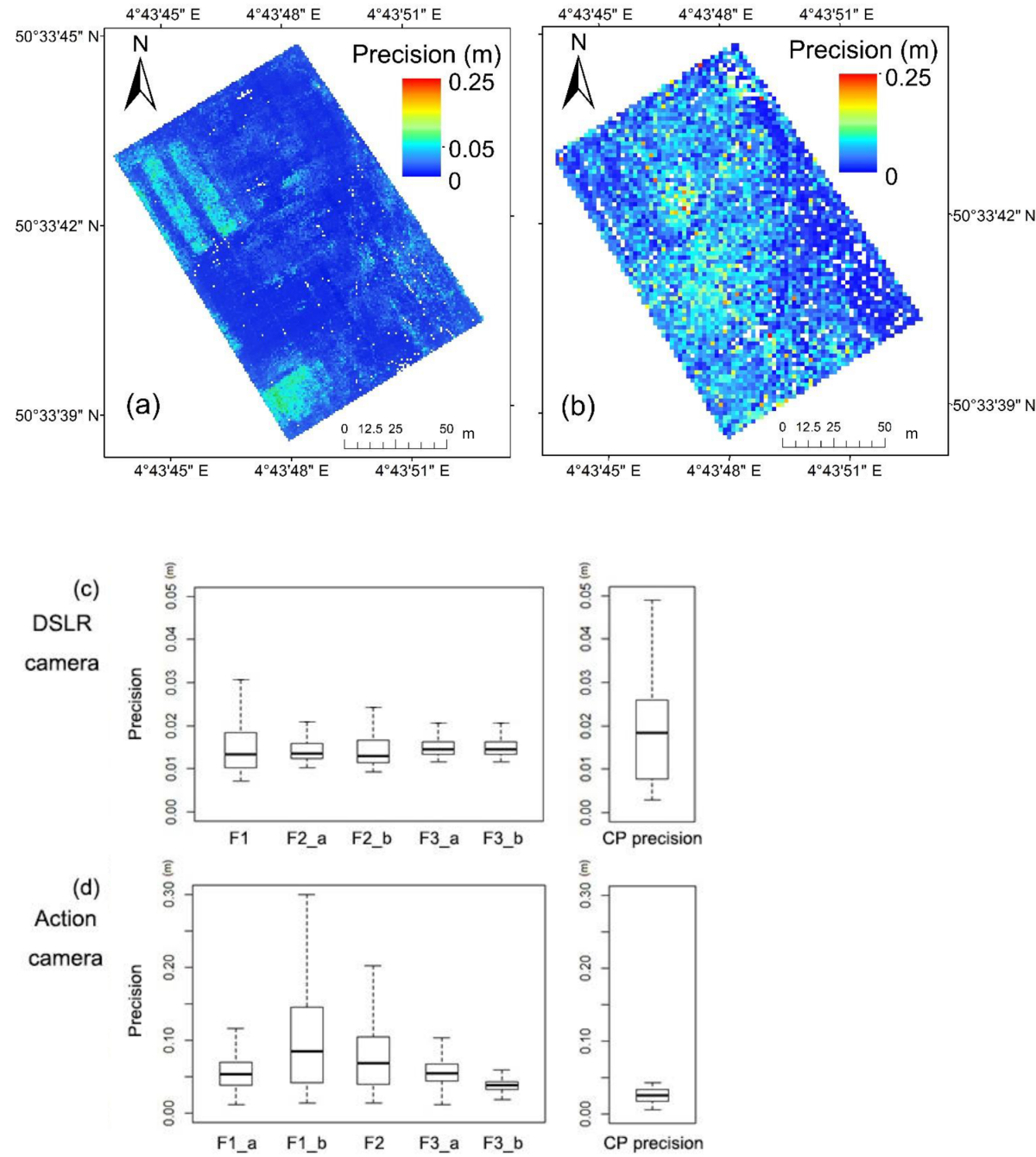

Figure 5. Precision maps derived from Monte Carlo simulation. (a) MC map of DSLR camera, dataset: F3_a. (b) MC map of action camera, dataset: F2_b. (c) Box plots of MC precision of DSLR surveys (area of interest). (d) Box plots of MC precision of action camera surveys (area of interest).

error surface (based on MC precision). Note that due to the different flight altitudes (35 and $20 \mathrm{~m}$ for the DSLR and action camera, respectively), the DoD thresholds were similar for both cameras. Similar change detection can be obtained using the constant DoD for both cameras (Fig. 6a). As reported above, the MC precision showed smaller uncertainties for the DSLR dataset than for the action camera dataset, leading to different levels of detection. The zoomed-in area shows the detail of the surface changes along a profile and its DoD threshold (Fig. 6b). The DSLR camera provided much more detail than the profile generated by the action camera. Nevertheless, a significant surface change could be detected for both approaches when using the $\mathrm{LoD}_{\min }$ threshold. We assessed the volume changes over the area of interest while considering the LoD thresholds. Regarding the PPK solution, the volume estimations of the two camera datasets had significant differences due to the existing bias. For the
$P P K+1 G C P$ datasets, when using a spatially uniform (i.e., constant average) LoD, the DSLR dataset resulted in a total volume lowering of $8.17 \pm 2.70 \mathrm{~m}^{3}$, while a volume increase of $175.50 \pm 76.33 \mathrm{~m}^{3}$ was detected due to changes in bulk density and the construction of ridges. The action camera dataset evaluated the volume decrease at $6.16 \pm 2.36 \mathrm{~m}^{3}$, while $191.77 \pm 99.18 \mathrm{~m}^{3}$ accumulated (Fig. 6c). When using MC LoD, the estimated volume of changes was $155.96 \pm$ $35.05 \mathrm{~m}^{3}$ for the DSLR camera and $92.60 \pm 66.5 \mathrm{~m}^{3}$ for the action camera. Both the DSLR and action camera obtained similar estimations using constant LoD. The MC LoD for DSLR datasets resulted in more significant surface changes than those obtained for the action camera dataset. 
(a)

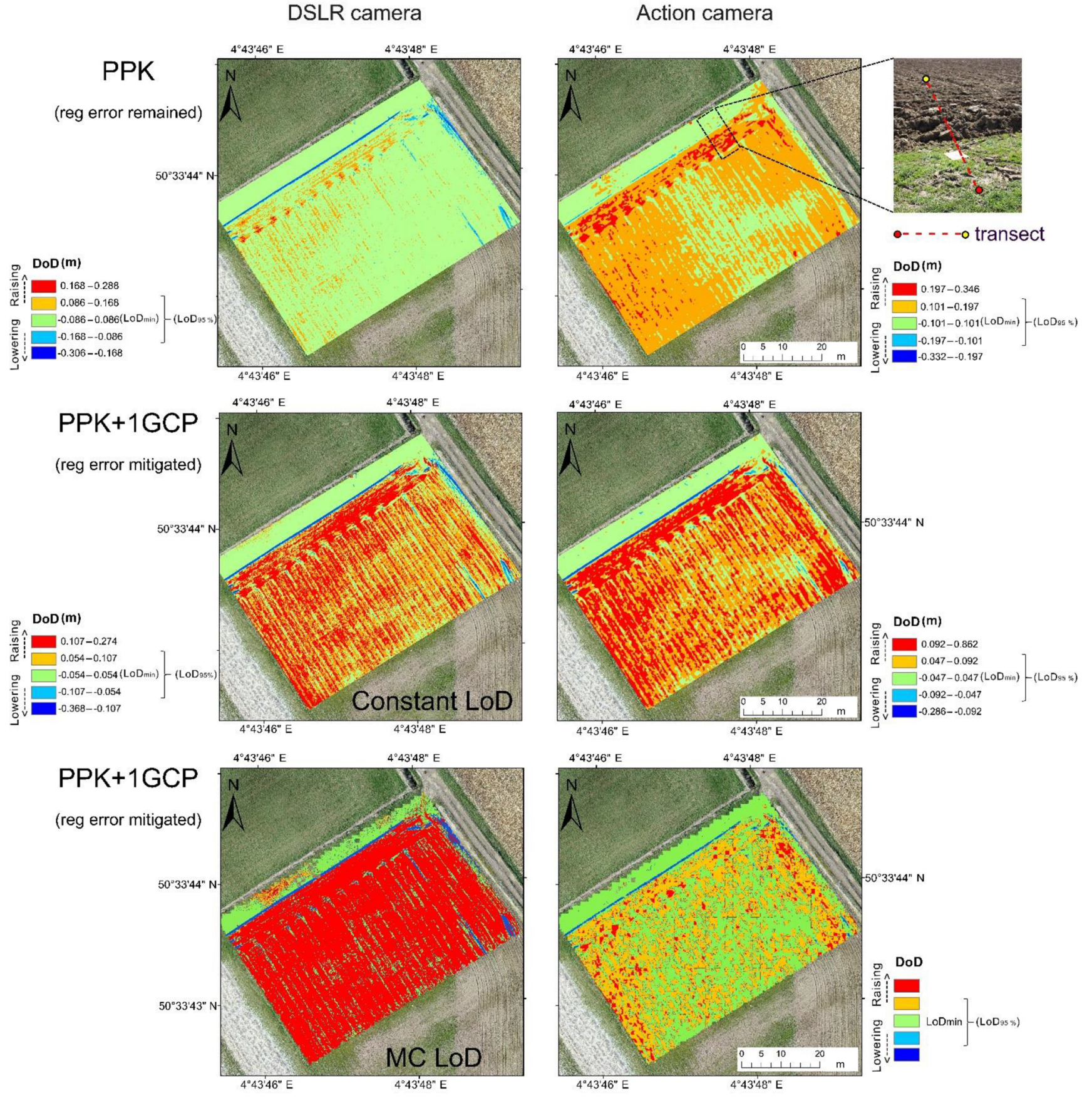

Figure 6.

\section{Discussion}

\subsection{Accuracy and precision of PPK solution in direct georeferencing}

The PPK direct georeferencing provided centimeter-level accuracy and precision during a $14 \mathrm{~d}$ monitoring campaign during which light conditions, image quality and GPS satellite constellation changed. This indicates that direct georeferencing with accurate positioning is capable of replacing the conventional ground control method and allows for the acquisition of robust centimetric HRT data. As already indicated by many studies, a single onboard GPS provides meter-level accuracy (Turner et al., 2012a). The quality of GCP-based georeferencing depends on the number and distribution of GCPs (Sanz-Ablanedo et al., 2018). The accuracy can be 
(b)
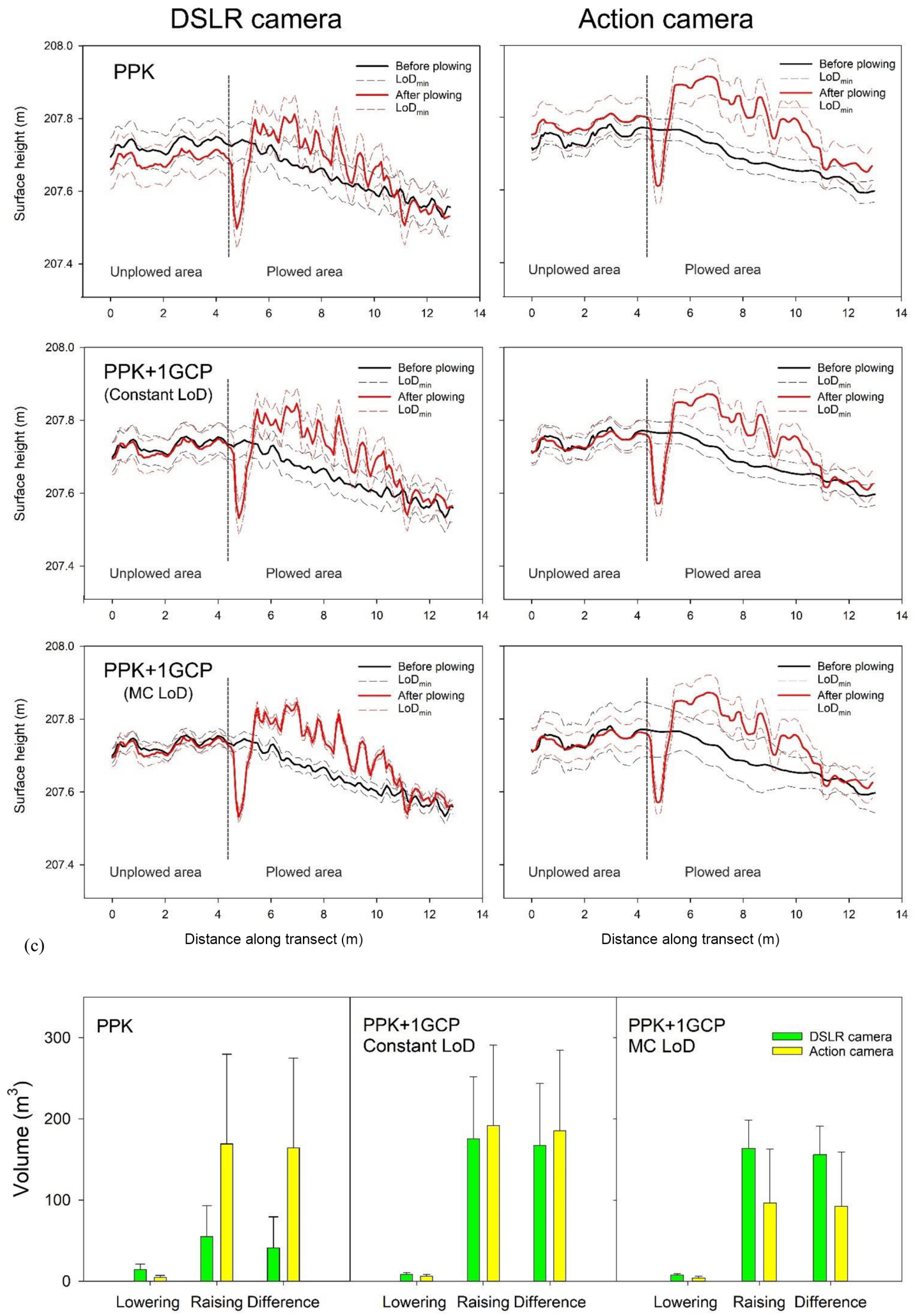

Figure 6. Change detection based on DoD (datasets: F2, F3_a of DSLR and action camera surveys). (a) Surface change map. (b) Height profiles sampled at an identical location from the corresponding DSMs before and after plowing. Line graph shows height profiles along the sample transect ( $X$ axis: position along the transect, $Y$ axis: surface height). (c) Volumetric sediment budget. 
improved by introducing additional and more densely distributed GCPs, which induces a trade-off between survey time and the quality of surface reconstruction (Eltner et al., 2016; Smith et al., 2016). Areas with poor distributions of GCPs or lower control precision could be vulnerable to systematic errors (James et al., 2017). For example, in remote glacier studies (Kraaijenbrink et al., 2016), GCPs can generally only be located at the glacier periphery, which is unfavorable for internal accuracy. In contrast, precise direct georeferencing of aerial surveys (kinematic GNSS) provides an evenly distributed control framework as each image can be regarded as a control point. Figure A2 exhibits the planimetric image residuals between the original image positions and the optimized positions after the BBA process. This shows that the image residuals were evenly distributed and had standard deviations of only a few centimeters, indicating there was little bias during the image georeferencing process. The DSLR images had smaller SD of positional residuals than those of the action camera images, indicating that the action camera images had higher random error regarding the BBA process.

In this study, our experiments showed that a high-quality GNSS receiver mounted on an aluminum plate that is positioned as far as possible from the UAV electronics can provide reliable accuracy and precision in positioning camera locations. Initial tests showed that the GPS data quality is very vulnerable to interferences from the UAV motors and electronics, and special attention should be given to shielding. The PPK positioning (without GCPs) of camera positions was shown to provide the same level of accuracy and precision as a GCP solution in our case. Nevertheless, there might be biases in the PPK GNSS position estimation due to false solutions that can remain undetected (e.g., false fix in resolving ambiguities). An approach to detecting this is to check the accordance between fix-and-hold and continuous resolution in integer ambiguity in RTKLib). Implementing one GCP did improve the results in our study: on average the addition of a single GCP slightly reduced the overall RMSEs. Given that it is difficult to assess the quality of the PPK solution without independent observation, we recommend that using one GCP (or one single fixed point throughout the monitoring) provides a robust way to detect perturbations of the GPS signal. Forlani et al. (2018) balanced the advantage of an RTK-PPK versus a GCP solution and reported that for the $R T K+1 G C P$ configuration, the vertical bias was greatly reduced. It should be noted that applying one GCP only moves the overall project to the approximate location without internal georeferencing.

\subsection{DSLR versus action camera}

As for the cameras we used in this study, the main differences were related to the focal length, image resolution and quality. The action camera with shorter focal length $(2.92 \mathrm{~mm})$ provides a larger field of view (diagonal FOV: $149.2^{\circ}$ ) but is characterized by radial lens distortions. The vertical errors derived from 16 individual check points were all below $0.07 \mathrm{~m}$, indicating that the "doming" effect can be greatly eliminated or mitigated due to the dense and precise control of camera positions. The DSLR camera, due to a larger APS-C-sized imager, higher focal length and higher resolution, together with the complete control of the ISO, shutter speed and aperture settings, produced much less noise and better overall picture quality. These differences led to better GSD and image contrast. We observed that this assisted greatly in recognizing and matching tie points. For instance, at $45 \mathrm{~m}$ of flight height, the DSLR dataset has a higher tie point density (mean: 213.5 points $\mathrm{m}^{-2}$ ) than the action camera (mean: 12.1 points $\mathrm{m}^{-2}$ ), and the detailed images help improve finding and matching tie points.

To visualize the two camera setup outputs and assess the potential of soil roughness measurement in different surface types, we derived two representative transects (Fig. 6b). Due to a higher GSD, the DSLR-derived data showed abundant and sharp details, while data from the action camera were relatively smooth. It should be noted that due to the large FOV, the action camera required a flight plan that was much less dense than for the DSLR camera (about half), indicating that a much larger area (about double) could be surveyed in the same time. However, this larger spatial coverage comes at the cost of ground resolution. A lower distance between the camera sensor and the surface is required for the action camera to obtain the same GSD as the DSLR camera (for the GoPro and EOS cameras used in this study, the flight height ratio to obtain the same GSD equals $1: 3.5$, and the consumed time ratio was ca. $1: 1.5$ ). For the design and practical implementation of UAV surveys, it is crucial to take the sensor weight and size into account, as well as the payload and endurance of the UAV platform. We found that with a light, small, highly portable and low-cost UAV equipped with a very simple camera and RTK-PPK GPS system, very good results in terms of accuracy and precision are possible (RMSE of ca. 1 pixel). In addition, taking advantage of the large FOV of the compact action camera, it is feasible to cover more area but at the cost of GSD and accuracy.

\subsection{Precision estimates}

We observed some inconsistencies between the MC-derived precision and CP-derived precision estimates. The observational precision for the DSLR dataset was slightly worse than that obtained from the MC estimates. We attribute this to the fact that $\mathrm{CP}$ itself can be regarded as a key feature that is easy to recognize in the BBA process. In addition, the observational precision reflects all sources of uncertainty, while the MC only considered the camera position. In contrast, the $\mathrm{MC}$ precision of the action camera dataset was much lower than the $\mathrm{CP}$ precision, which results from the high radial distortion feature of the high-FOV lens and the lower GSD. 
To identify which factor (high GSD or low FOV) controls the precision estimates, we preprocessed the images using two methods: (i) down-sample the DSLR images to have the same GSD as action camera images, and (ii) clip the action camera images to have the same FOV as DSLR images (for that, we implemented an additional flight mission for the action camera using a denser flight path). Precision maps were then generated using Monte Carlo simulation (Fig. A3). With a lower GSD, the precision pattern for the DSLR dataset remained but showed increased uncertainties. In contrast, the clipped low-FOV action camera images revealed a clear spatial pattern for the precision estimates. Based on this analysis, we suggest that a higher GSD increased the robustness of the tie point matching and hence improved the precision. The large FOV of the action camera, enabling wide imaging angles to a single tie point, may to some extent compensate for the difficulties in the identification of key features due to the lower GSD, at least if appropriate model calibrations are introduced in the bundle adjustment. It should be noted that the radial distortion induced by the fish-eye lens is more severe on the edges of the images. This increases the uncertainties in tie point orientation and may explain the higher magnitude of tie point uncertainties (Fig. 5b).

\subsection{Surface change detection}

Using an average RMSE to estimate the registration error resulted in poor estimates of surface change. This was related to the fact that the PPK solution provided results with substantial bias in the $Z$ direction for a few flights. The repeatability assessment showed that the use of a flight-specific registration error based on one GCP could remove the bias. Furthermore, this study showed that the approach is repeatable as both UAV-camera setups resulted in a similar estimation of 3-D surface changes. To obtain a robust change detection, it is crucial to set a proper uncertainty threshold (LoD). Our results indicated that the approach to estimate the LoD (i.e., MC-based versus CP-based) substantially affect the results, particularly for small-scale, high-resolution applications (i.e., that require centimetric precision). It is also important to understand the effect of different types of surfaces on the SfM output, particularly in a region with a "complex" surface, e.g., vegetation area, rough objects and a surface with few key features. Vegetation has long been recognized as a source of error in photogrammetry (Lane et al., 2000; Messinger et al., 2016) due to the clustering of leaves, wind-caused movement and illumination change, and this increased the complexity of the imagery, leading to difficulties in isolating tie points (Harwin and Lucieer, 2012). Applying a spatially explicit error threshold in topographic change detection can help improve the reliability and sensitivity.

Our study demonstrates that the PPK positioning is a robust solution for monitoring surface change and estimating sediment budgets at very high spatial and temporal resolution. This technique can be very advantageous when it comes to monitoring large areas that are poorly accessible or require repeated surveying (Clapuyt et al., 2017; Eltner et al., 2016). A relatively cheap RTK-PPK-enabled micro-UAV (small form $25 \times 25 \mathrm{~cm}$, weight $1.4 \mathrm{~kg}$, autonomy $15 \mathrm{~min}$ ) provided similar accuracy and repeatability as a professional multirotor UAV system (large form $80 \times 80 \mathrm{~cm}$, weight $4.5 \mathrm{~kg}$, autonomy $15 \mathrm{~min}$ ). Based on our analysis, we suggest that using a micro-drone-action camera setup is suitable for largescale monitoring (e.g., gully erosion, landslides, glaciers, etc.) when a high GSD is not required. When considering a scene's 3-D geometry, the high FOV also assists in recording features exposed along vertical facades (e.g., vertical cliff face) from nadir-view photogrammetry. Furthermore, in countries with strict UAV regulations and/or inaccessible regions (e.g., mountains) a lightweight system can be more easily transported in the field than a large UAV system. The DSLR camera setup can be used when high resolution is needed, for example for soil roughness assessment, sheet and tillage erosion, solifluction, and riverbank erosion. Finally, a key step in PPK positioning is to obtain GPS data from a stationary base station. In this study, we used an internetenabled system to geolocate the base station for each flight. For areas where internet is absent or unreliable and long-term monitoring is required, we suggest setting up a permanent reference point that can be used to position a local base station (e.g., a concrete pole).

\section{Conclusions}

The UAV-SfM framework is increasingly used in geomorphology to accurately capture the Earth's surface. Our study showed that the application of PPK (post-processing kinematic) in direct georeferencing can provide centimeter-level accuracy and precision, which results in a greatly improved field survey efficiency. Furthermore, it is a robust method that was demonstrated to be repeatable among multiple dates and surveys. We investigated the positional accuracy and the repeatability of DSMs by repeating the same flight plans. The PPK solution had a similar accuracy (MAE: ca. $0.02 \mathrm{~m}$, RMSE: ca. $0.03 \mathrm{~m}$ ) as the traditional approach using georeferencing based on GCPs. Nevertheless, some flights were characterized by a vertical shift that could be mitigated using a single GCP. We also evaluated two UAV-camera setups (with differences in UAV size and weight, portability, camera focal length, resolution, and sensor quality) and showed that the tie point uncertainties are very different. Nevertheless, the DSM reconstruction and surface change detection based on a DSLR and action camera were reproducible: the main difference lies in the level of detail of the surface representations. Using low-altitude flights $(<45 \mathrm{~m})$ it is possible to detect surface change using a PPK-SfM workflow with a threshold below $5 \mathrm{~cm}$, even with a low-cost action camera. Precision estimates are critical to assess significant changes between two surfaces. We evaluated different methods to es- 
timate precision and registration errors and found that Monte Carlo simulations (James et al., 2017), in which the camera position uncertainty is considered, provide a robust way to estimate spatially explicit LoD thresholds for low-FOV cameras. Overall, the PPK-SfM workflow overcomes some of the main limitations of GCPs and provides a high-precision and high-efficiency solution in surveying and geomorphological applications.
Data availability. All data used and produced through this study are available upon request. 
Appendix A
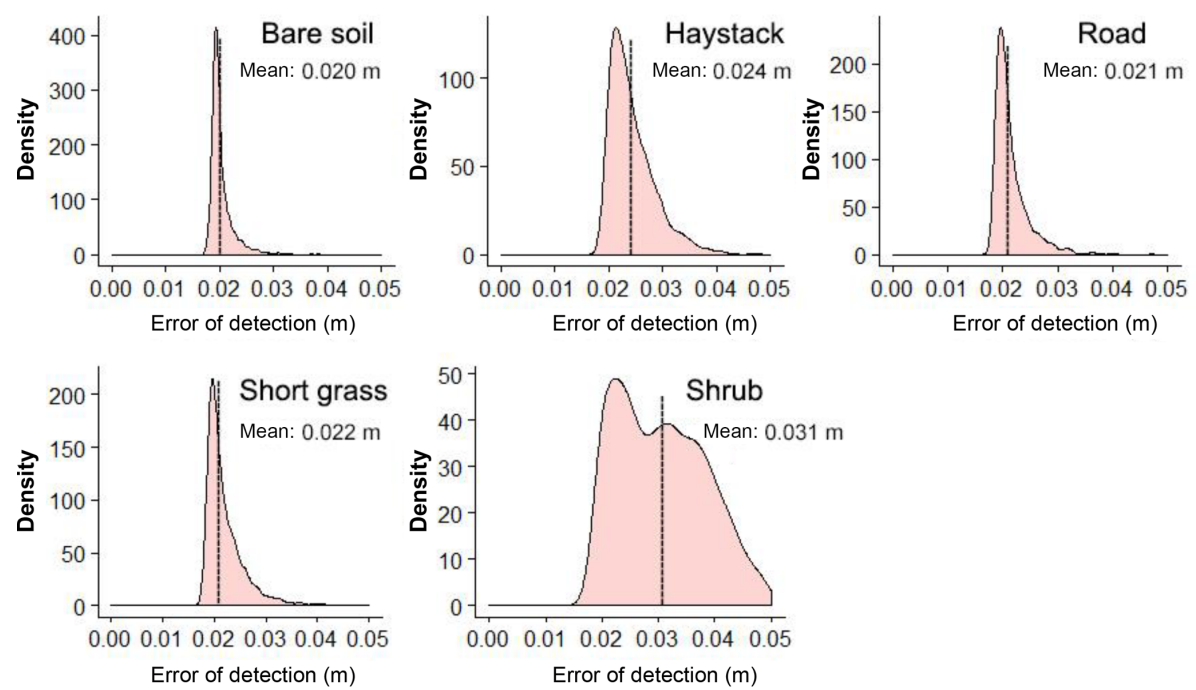

Figure A1. Distribution of the propagated error derived from Monte Carlo simulation (datasets: F3_a and F3_b of DSLR surveys; surface classification shown in Fig. 1c).
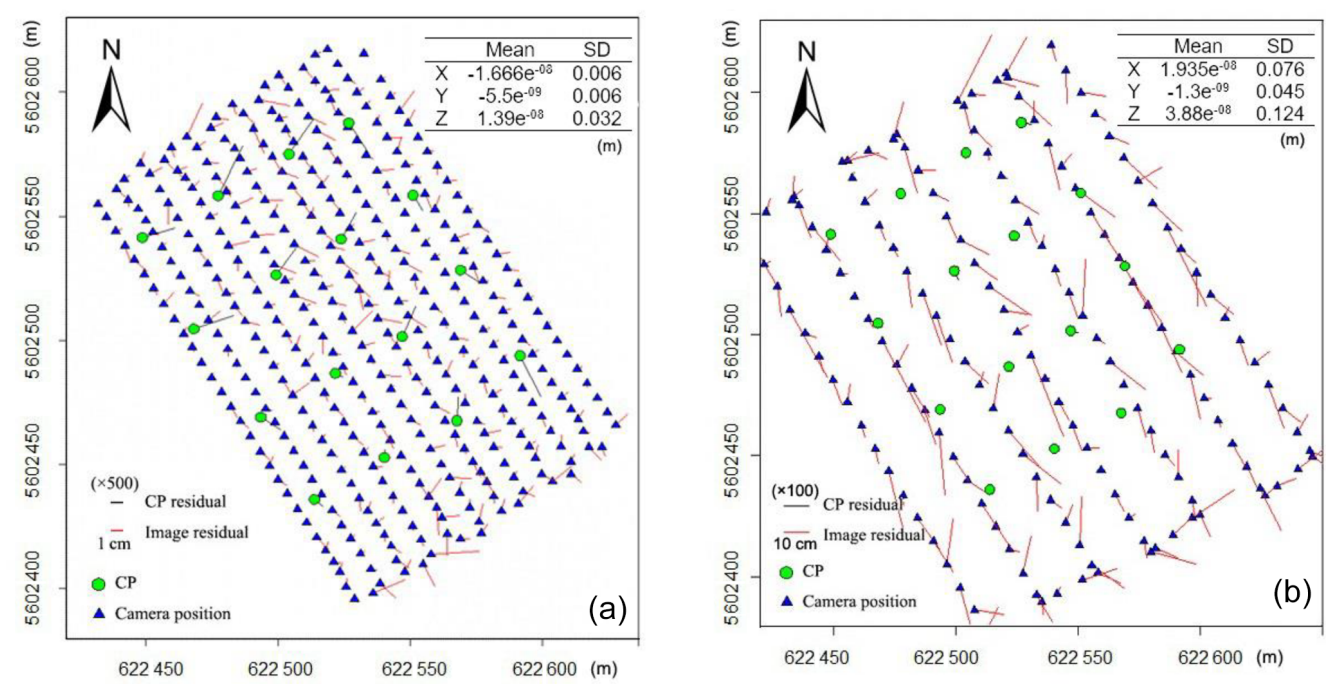

Figure A2. Residuals on the images and CPs in planimetric view. Vectors give the horizontal residual component magnified by $\times 500$ for the DSLR survey (a) and $\times 100$ for the action camera survey (b). Inset: mean value and standard deviation of the image residuals. 

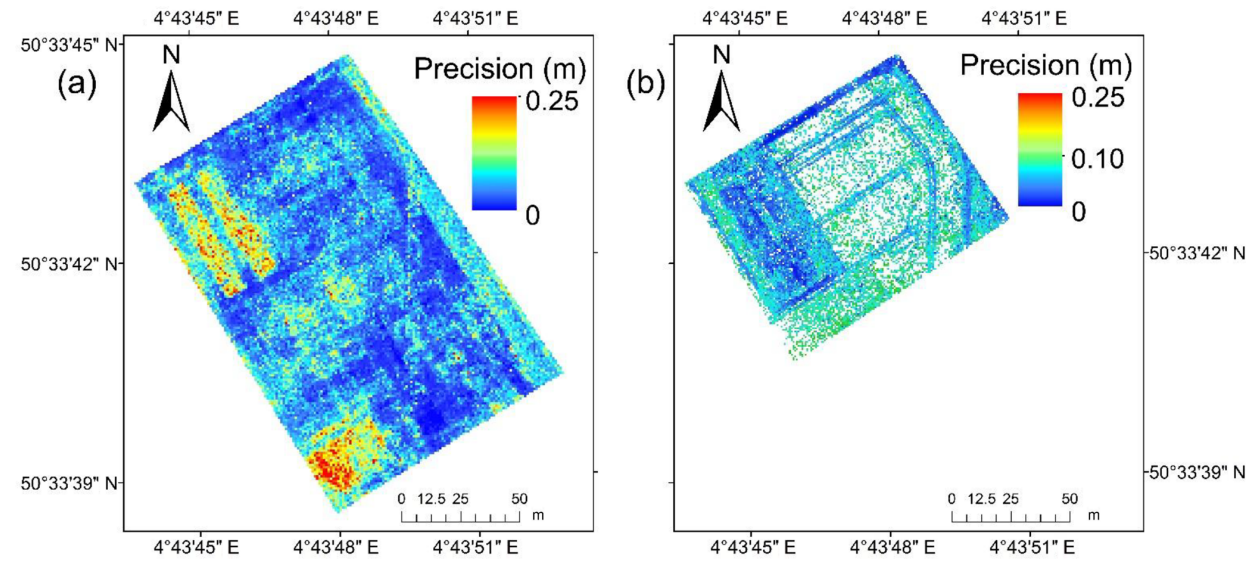

Figure A3. Monte Carlo Precision maps. (a) Dataset: down-sampled DSLR images with equal GSD as action camera images. (b) Dataset: clipped action camera images with equal FOV. Note: the additional action camera flight mission (right) was conducted 1 year later and the surface had slightly changed, but there was a spatial pattern. 
Author contributions. HZ, EA and KVO designed the study and contributed to fieldwork. $\mathrm{HZ}$ and KVO performed data analysis. All authors offered advice on data analysis and contributed to paper preparation.

Competing interests. The authors declare that they have no conflict of interest.

Acknowledgements. We thank Mike James, Joan-Cristian Padró and anonymous reviewers for their constructive feedback that improved the paper. We are also thankful to Richard Gloaguen and the editors for their constructive comments and careful review.

Financial support. This research has been supported by the China Scholarship Council (grant no. 201706300034) and the BELSPO Stereo Programme (RAPAS Project) (grant no. SR/00/328).

Review statement. This paper was edited by Richard Gloaguen and reviewed by Mike James, Joan-Cristian Padró, and one anonymous referee.

\section{References}

Brasington, J., Rumsby, B. T., and McVey, R. A.: Monitoring and modelling morphological change in a braided gravelbed river using high resolution GPS-based survey, Earth Surf. Proc. Land., 25, 973-990, https://doi.org/10.1002/10969837(200008)25:9<973::AID-ESP111>3.0.CO;2-Y, 2000.

Brasington, J., Langham, J., and Rumsby, B.: Methodological sensitivity of morphometric estimates of coarse fluvial sediment transport, Geomorphology, 53, 299-316, https://doi.org/10.1016/S0169-555X(02)00320-3, 2003.

Candiago, S., Remondino, F., De Giglio, M., Dubbini, M., and Gattelli, M.: Evaluating multispectral images and vegetation indices for precision farming applications from UAV images, Remote Sens., 7, 4026-4047, https://doi.org/10.3390/rs70404026, 2015.

Clapuyt, F., Vanacker, V., and Van Oost, K.: Reproducibility of UAV-based earth topography reconstructions based on Structure-from-Motion algorithms, Geomorphology, 260, 4-15, https://doi.org/10.1016/j.geomorph.2015.05.011, 2016.

Clapuyt, F., Vanacker, V., Schlunegger, F., and Van Oost, K.: Unravelling earth flow dynamics with 3-D time series derived from UAV-SfM models, Earth Surf. Dynam., 5, 791-806, https://doi.org/10.5194/esurf-5-791-2017, 2017.

d'Oleire-Oltmanns, S., Marzolff, I., Peter, K., and Ries, J.: Unmanned aerial vehicle (UAV) for monitoring soil erosion in Morocco, Remote Sens., 4, 3390-3416, 2012.

Duró, G., Crosato, A., Kleinhans, M. G., and Uijttewaal, W. S. J.: Bank erosion processes measured with UAV-SfM along complex banklines of a straight mid-sized river reach, Earth Surf. Dynam., 6, 933-953, https://doi.org/10.5194/esurf-6-933-2018, 2018.

Eker, R., Aydın, A., and Hübl, J.: Unmanned aerial vehicle (UAV)based monitoring of a landslide: Gallenzerkogel landslide (Ybbs-
Lower Austria) case study, Environ. Monit. Assess., 190, 28, https://doi.org/10.1007/s10661-017-6402-8, 2018.

Eltner, A. and Schneider, D.: Analysis of Different Methods for 3-D Reconstruction of Natural Surfaces from ParallelAxes UAV Images, Photogramm. Rec., 30, 279-299, https://doi.org/10.1111/phor.12115, 2015.

Eltner, A., Baumgart, P., Maas, H., and Faust, D.: Multi-temporal UAV data for automatic measurement of rill and interrill erosion on loess soil, Earth Surf. Proc. Land., 40, 741-755, 2015.

Eltner, A., Kaiser, A., Castillo, C., Rock, G., Neugirg, F., and Abellán, A.: Image-based surface reconstruction in geomorphometry - merits, limits and developments, Earth Surf. Dynam., 4, 359389, https://doi.org/10.5194/esurf-4-359-2016, 2016.

Eltner, A., Kaiser, A., Abellan, A., and Schindewolf, M.: Time lapse structure-from-motion photogrammetry for continuous geomorphic monitoring, Earth Surf. Proc. Land., 42, 2240-2253, https://doi.org/10.1002/esp.4178, 2017.

Fazeli, H., Samadzadegan, F., and Dadrasjavan, F.: Evaluating the potential of RTK-UAV for automatic point cloud generation in 3-D rapid mapping, Int. Arch. Photogramm., 41, 221-226, https://doi.org/10.5194/isprsarchives-XLI-B6-221-2016, 2016.

Forlani, G., Dall'Asta, E., Diotri, F., di Cella, U. M., Roncella, R., and Santise, M.: Quality assessment of DSMs produced from UAV flights georeferenced with on-board RTK positioning, Remote Sens., 10, 1-22, https://doi.org/10.3390/rs10020311, 2018

Fuller, I. C., Large, A. R. G., Charlton, M. E., Heritage, G. L., and Milan, D. J.: Reach-scale sediment transfers: An evaluation of two morphological budgeting approaches, Earth Surf. Proc. Land., 28, 889-903, https://doi.org/10.1002/esp.1011, 2003.

Gerke, M. and Przybilla, H.-J.: Accuracy Analysis of Photogrammetric UAV Image Blocks: Influence of Onboard RTK-GNSS and Cross Flight Patterns, Photogramm. Fernerkun., 2016, 17 30, https://doi.org/10.1127/pfg/2016/0284, 2016.

Glendell, M., McShane, G., Farrow, L., James, M. R., Quinton, J., Anderson, K., Evans, M., Benaud, P., Rawlins, B., Morgan, D., Jones, L., Kirkham, M., DeBell, L., Quine, T. A., Lark, M., Rickson, J., and Brazier, R. E.: Testing the utility of structure-frommotion photogrammetry reconstructions using small unmanned aerial vehicles and ground photography to estimate the extent of upland soil erosion, Earth Surf. Proc. Land., 42, 1860-1871, https://doi.org/10.1002/esp.4142, 2017.

Grayson, B., Penna, N. T., Mills, J. P., and Grant, D. S.: GPS precise point positioning for UAV photogrammetry, Photogramm. Rec., 33, 427-447, 2018.

Harwin, S. and Lucieer, A.: Assessing the accuracy of georeferenced point clouds produced via multi-view stereopsis from Unmanned Aerial Vehicle (UAV) imagery, Remote Sens., 4, 15731599, https://doi.org/10.3390/rs4061573, 2012.

Hawkins, B. S.: Using a drone and photogrammetry software to create orthomosaic images and 3-D models of aircraft accident sites, in: ISASI 2016 Seminar, 17-20 October 2016, Reykjavik, Iceland, 1-26, 2016.

Hemmelder, S., Marra, W., Markies, H., and De Jong, S. M.: Monitoring river morphology \& bank erosion using UAV imagery - A case study of the river Buëch, HautesAlpes, France, Int. J. Appl. Earth Obs. Geoinf., 73, 428-437, https://doi.org/10.1016/j.jag.2018.07.016, 2018.

James, M. R. and Robson, S.: Straightforward reconstruction of 3-D surfaces and topography with a camera: Accuracy and 
geoscience application, J. Geophys. Res.-Earth, 117, 1-17, https://doi.org/10.1029/2011JF002289, 2012.

James, M. R. and Robson, S.: Mitigating systematic error in topographic models derived from UAV and ground-based image networks, Earth Surf. Proc. Land., 39, 1413-1420, https://doi.org/10.1002/esp.3609, 2014.

James, M. R., Robson, S., and Smith, M. W.: 3-D uncertaintybased topographic change detection with structure-from-motion photogrammetry: precision maps for ground control and directly georeferenced surveys, Earth Surf. Proc. Land., 42, 1769-1788, https://doi.org/10.1002/esp.4125, 2017.

Kraaijenbrink, P. D. A., Shea, J. M., Pellicciotti, F., De Jong, S. M., and Immerzeel, W. W.: Object-based analysis of unmanned aerial vehicle imagery to map and characterise surface features on a debris-covered glacier, Remote Sens. Environ., 186, 581595,2016

Lane, S. N., James, T. D., and Crowell, M. D.: Application of digital photogrammetry to complex topography for geomorphological research, Photogramm. Rec., 16, 793-821, https://doi.org/10.1111/0031-868X.00152, 2000.

Messinger, M., Asner, G. P., and Silman, M.: Rapid assessments of amazon forest structure and biomass using small unmanned aerial systems, Remote Sens., 8, 1-15, https://doi.org/10.3390/rs8080615, 2016.

Micheletti, N., Chandler, J. H., and Lane, S. N.: Investigating the geomorphological potential of freely available and accessible structure-from-motion photogrammetry using a smartphone, Earth Surf. Proc. Land., 40, 473-486, https://doi.org/10.1002/esp.3648, 2015.

Mosbrucker, A. R., Major, J. J., Spicer, K. R., and Pitlick, J.: Camera system considerations for geomorphic applications of SfM photogrammetry, Earth Surf. Proc. Land., 42, 969-986, https://doi.org/10.1002/esp.4066, 2017.

Ouédraogo, M. M., Degré, A., Debouche, C., and Lisein, J.: The evaluation of unmanned aerial system-based photogrammetry and terrestrial laser scanning to generate DEMs of agricultural watersheds, Geomorphology, 214, 339-355, https://doi.org/10.1016/j.geomorph.2014.02.016, 2014.

Padró, J.-C., Muñoz, F.-J., Planas, J., and Pons, X.: Comparison of four UAV georeferencing methods for environmental monitoring purposes focusing on the combined use with airborne and satellite remote sensing platforms, Int. J. Appl. Earth Obs. Geoinf., 75, 130-140, 2019.

Passalacqua, P., Belmont, P., Staley, D. M., Simley, J. D., Arrowsmith, J. R., Bode, C. A., Crosby, C., DeLong, S. B., Glenn, N. F., Kelly, S. A., Lague, D., Sangireddy, H., Schaffrath, K., Tarboton, D. G., Wasklewicz, T., and Wheaton, J. M.: Analyzing high resolution topography for advancing the understanding of mass and energy transfer through landscapes: A review, Earth-Sci. Rev., 148, 174-193, https://doi.org/10.1016/j.earscirev.2015.05.012, 2015.

Pineux, N., Lisein, J., Swerts, G., Bielders, C. L., Lejeune, P., Colinet, G., and Degré, A.: Can DEM time series produced by UAV be used to quantify diffuse erosion in an agricultural watershed?, Geomorphology, 280, 122-136, https://doi.org/10.1016/j.geomorph.2016.12.003, 2017.

Rosnell, T. and Honkavaara, E.: Point cloud generation from aerial image data acquired by a quadrocopter type micro unmanned aerial vehicle and a digital still camera, Sensors, 12, 453-480, https://doi.org/10.3390/s120100453, 2012.

Rossini, M., Di Mauro, B., Garzonio, R., Baccolo, G., Cavallini, G., Mattavelli, M., De Amicis, M., and Colombo, R.: Rapid melting dynamics of an alpine glacier with repeated UAV photogrammetry, Geomorphology, 304, 159-172, https://doi.org/10.1016/j.geomorph.2017.12.039, 2018.

Sanz-Ablanedo, E., Chandler, J. H., and Wackrow, R.: Parameterising Internal Camera Geometry with Focusing Distance, Photogramm. Rec., 27, 210-226, https://doi.org/10.1111/j.14779730.2012.00677.x, 2012.

Sanz-Ablanedo, E., Chandler, J., Rodríguez-Pérez, J., Ordóñez, C., Sanz-Ablanedo, E., Chandler, J. H., Rodríguez-Pérez, J. R., and Ordóñez, C.: Accuracy of Unmanned Aerial Vehicle (UAV) and SfM Photogrammetry Survey as a Function of the Number and Location of Ground Control Points Used, Remote Sens., 10, 1606, https://doi.org/10.3390/RS10101606, 2018.

Smith, M. W., Carrivick, J. L., and Quincey, D. J.: Structure from motion photogrammetry in physical geography, Prog. Phys. Geogr., 40, 247-275, 2016.

Stöcker, C., Nex, F., Koeva, M., and Gerke, M.: Quality assessment of combined IMU/GNSS data for direct georeferencing in the context of UAV-based mapping, Int. Arch. Photogramm., 42, 355-361, https://doi.org/10.5194/isprs-archives-XLII-2-W6355-2017, 2017.

Takasu, T. and Yasuda, A.: Development of the low-cost RTKGPS receiver with an open source program package RTKLIB, in: International Symposium on GPS/GNSS, 4-6 November 2009, Jeju, Korea, 2009.

Tarolli, P.: High-resolution topography for understanding Earth surface processes: Opportunities and challenges, Geomorphology, 216, 295-312, https://doi.org/10.1016/j.geomorph.2014.03.008, 2014.

Triggs, B., McLauchlan, P. F., Hartley, R. I., and Fitzgibbon, A. W.: Bundle Adjustment - A Modern Synthesis, in Vision Algorithms: Theory and Practice, edited by: Triggs, B., Zisserman, A., and Szeliski, R., 298-372, Springer, Berlin, Heidelberg, 2000.

Turner, D., Lucieer, A., and Watson, C.: An automated technique for generating georectified mosaics from ultra-high resolution unmanned aerial vehicle (UAV) imagery, based on structure from motion (SfM) point clouds, Remote Sens., 4, 1392-1410, 2012a.

Turner, D., Lucieer, A., and Watson, C.: An automated technique for generating georectified mosaics from ultra-high resolution Unmanned Aerial Vehicle (UAV) imagery, based on Structure from Motion (SFM) point clouds, Remote Sens., 4, 1392-1410, https://doi.org/10.3390/rs4051392, 2012b.

Turner, D., Lucieer, A., and de Jong, S. M.: Time series analysis of landslide dynamics using an Unmanned Aerial Vehicle (UAV), Remote Sens., 7, 1736-1757, https://doi.org/10.3390/rs70201736, 2015.

Turner, I. L., Harley, M. D., and Drummond, C. D.: UAVs for coastal surveying, Coast. Eng., 114, 19-24, https://doi.org/10.1016/j.coastaleng.2016.03.011, 2016.

Uysal, M., Toprak, A. S., and Polat, N.: DEM generation with UAV Photogrammetry and accuracy analysis in Sahitler hill, Measurement, 73, 539-543, https://doi.org/10.1016/j.measurement.2015.06.010, 2015.

Wheaton, J. M., Brasington, J., Darby, S. E., and Sear, D. A.: Accounting for uncertainty in DEMs from repeat topographic sur- 
veys: Improved sediment budgets, Earth Surf. Proc. Land., 35, 136-156, https://doi.org/10.1002/esp.1886, 2010.

Wheaton, J. M., Brasington, J., Darby, S. E., Kasprak, A., Sear, D., and Vericat, D.: Morphodynamic signatures of braiding mechanisms as expressed through change in sediment storage in a gravel-bed river, J. Geophys. Res.-Earth, 118, 759-779, https://doi.org/10.1002/jgrf.20060, 2013. 